\title{
https://doi.org/10.46813/2020-130-064 \\ TRANSILLUMINATION OF THE DENSE PLASMA BARRIER BY THE STRONG BEAM OF ELECTROMAGNETIC WAVES: COMPUTER SIMULATION
}

\author{
B.R. Mykhailenko, I.O. Anisimov \\ Taras Shevchenko National University of Kyiv, Kyiv, Ukraine \\ E-mail: mihaylenko.bogdan12@gmail.com
}

Transillumination of a dense plasma barrier by a strong electromagnetic wave beam has been studied via simulation using the PIConGPU software package. Formation of the channel with reduced plasma density, predicted theoretically and detected experimentally, was observed. For a higher power incident beam, plasma turbulent dynamics has been observed, which leads to its "fast" transillumination. The simulation results didn't confirm the theoretically proposed mechanisms of "fast" transillumination.

PACS: 52.35.Mw, 52.38.Hb

\section{INTRODUCTION}

Linear hydrodynamic theory assumes that dense non-magnetized plasma (even in the absence of electron-ion collisions) is opaque to electromagnetic waves. However, further theoretical and experimental studies have shown that there are a number of mechanisms for this waves' penetration through barriers (barrier transillumination), their transfer through barriers in one or another way (quasi-transparency) and incident wave information transfer to the trans-barrier area (information transparency) [1].

Such problems are important because real plasma is inhomogeneous almost always and often contains wave barriers. Therefore, there is a problem of waves` penetration inside and through the barriers, and, in particular, the transmission of information carried by waves, through the barriers. An example of such problems is radio communication with the spacecraft during its return to the Earth. When the spacecraft passes through the lower layers of the atmosphere, a dense plasma layer is formed in front of it, and radio communication can be blocked. The same range of problems includes the radio signals propagation from the Earth's surface to the area above the maximum density of ionosphere plasma at the low frequencies (this phenomenon was observed in the satellite experiments). Another possible application is microwave plasma diagnostics via raying, where the discussed effects can distort the result.

One of the possible transillumination mechanisms is the plasma barrier destruction by plasma displacing from some area or its redistribution by a field of an incident strong electromagnetic wave. The study of such nonlinear effects becomes even more relevant in connection with the problem of the laser-based inertial confinement fusion, as the corresponding experiments failed precisely due to the plasma displacement from the target caused by its irradiation non-uniformity [2].

Several possible mechanisms for the plasma barriers`destruction by an incident electromagnetic wave were proposed theoretically.

-A waveguide channel formation in the collisionless plasma due to the ponderomotive force caused by the inhomogeneous wave field [3]. As a result, plasma is pushed out of the strong electromagnetic field area, its density is reduced in the wave beam propagation path, a self-sustaining waveguide channel in the barrier is formed and the wave penetrates to the trans-barrier area.

- A waveguide channel formation in the plasma with the substantial electron collisions induced by heating nonlinearity caused by a Langmuir wave parametric excitation. With the normal incidence of a plane-polarized electromagnetic wave on the plasma layer, the Langmuir waves excited via parametric mechanism propagate in the direction of the incident wave electric field, i.e., their wave vector lies in the barrier plane. These waves damp effectively due to the electron collisions with heavy particles. As a result the electrons' temperature is increased and their density decreases accordingly. Consequently, the selfsustaining waveguide channel is formed [4].

- Periodic plasma density redistribution due to the standing electromagnetic wave field. With a normal incidence of an electromagnetic wave on a supercritical plasma layer with sharp boundaries, taking into account the ponderomotive force from the standing wave field leads to the possibility of the plasma density redistribution along the wave propagation direction. The result may be a spatially periodic (with a period of the order of the vacuum wavelength) plasma density distribution, and the plasma layer becomes partially transparent [5].

- Periodic plasma density redistribution due to the parametric Langmuir wave excitation. As mentioned, an electromagnetic wave normal incidence on plasma can be accompanied with the parametric Langmuir waves' excitation. The modulation instability of the Langmuir waves leads to the formation of the plasma density layers due to the ponderomotive force. As a result plasma dielectric permittivity changes its sign periodically in space. If the inhomogeneity spatial period is much smaller than the electromagnetic wave length, plasma becomes transparent. Then stratification 
is repeated in subsequent skin layers, and the wave penetrates deeper into the barrier [6]. This mechanism is realized at the initial stage of the limited crosssection wave beams' interaction with plasma, when the plasma density macroscopic redistribution effects have no enough time for development [3].

The first two mechanisms that lead to the channel formation in the barrier are known as the "slow" transillumination, and the last two ones that involve the density redistribution on a much smaller scale - as "fast" transillumination.

Plasma barriers' transillumination for electromagnetic waves was experimentally studied in [7-11]. The experiments were performed in decaying plasma $[4,7,9,10]$ or plasma that was formed by a high-frequency field with time-decreasing intensity [8, 11]. Therefore, both the density and the width of the plasma barrier decreased over time until it disappeared. As the incident wave power increased above some critical value, the wave behind the barrier appeared before it decayed. This critical value increased with the increase of the barrier supercriticality (the ratio of the maximum plasma density in the barrier to the critical plasma density at the incident wave frequency).

The authors of $[8,11]$ linked the detected transillumination with the plasma stratification as a result of the Langmuir waves parametric excitation by an incident electromagnetic wave and further modulation instability development $[3,6]$. This conclusion is supported by the dependence of the barrier transillumination for a low-power signal wave by a high-power wave on the mutual orientation of their polarization planes [8]: transillumination takes place if electric fields of both waves are parallel, and it is absent when they are mutually perpendicular.

The results of $[4,7,9-10]$, according to their authors, are better consistent with the waveguide channel formation mechanism caused by heating of the electron component of the plasma in the barrier [3]. In our opinion, such heating can be caused directly by the incident wave field in the skin layer and doesn't necessarily require Langmuir waves parametric excitation.

In [12] the effect of the incident electromagnetic wave polarization on the dense plasma barriers transillumination was studied. It has been shown that "fast" transillumination occurs for both linear and circular polarization of the strong incident wave. According to the authors, it suggests that the inhomogeneities formed in plasma, are at least twodimensional in contrast to the theoretical predictions $[3,6]$. Appearance of these inhomogeneities is hypothetically related to the plasma turbulent dynamics in the incident wave field.

Subsequent experiments $[13,14]$ using the diagnostic electromagnetic wave recorded depolarization effect and wave spectrum Doppler expansion and the emergence of satellites shifted to the ion-acoustic frequency. The diagnostic wave also allowed one to notice the existence of transillumination for some time $(1 \ldots 1.5 \mu \mathrm{s})$ after turning off the strong pumping wave. The emergence of low-frequency electric fields in plasma was recorded using a diagnostic electron beam. The authors attributed these results to the formation of three-dimensional smallscale inhomogeneities in plasma at the stage of its "fast" transillumination.

Thus, laboratory experiments confirmed the transillumination effect existence in two versions "fast" (before the waveguide channel formation) and "slow" transillumination. "Fast" transillumination is associated with plasma density redistribution. At the same time, the problem of the relationship between the experimental results and the proposed theoretical mechanisms remains unclear.

The purpose of this work is the computer simulation of the plasma barrier transillumination by a strong electromagnetic wave. Such simulation makes it possible to observe details of processes (for example, instantaneous spatial distributions of electron and ion density, electric field, etc.) that aren't available in a laboratory experiments. This, in turn, provides additional information for comparing the results of computer simulation with theoretical models. The preliminary simulation results are presented in this article.

\section{MODEL DESCRIPTION AND SIMULATION PACKAGE}

The PIConGPU software package [15] was used for simulation, which implements the algorithm of large particles in cells. The package is electromagnetic with plane geometry, it can describe relativistic effects. We used two-dimensional version. Electron-ion collisions aren't taken into account.

The normal incidence of a pulse of plane-polarized electromagnetic waves on a plane plasma layer in the absence of an external magnetic field was considered. The wave electric field lies in the simulation plane. The intensity of the incident wave beam is characterized by a Gaussian distribution in the $y$-direction and homogeneous in the $x$-direction (Fig. 1).

Electromagnetic field is absorbed at the boundaries of the simulation volume.

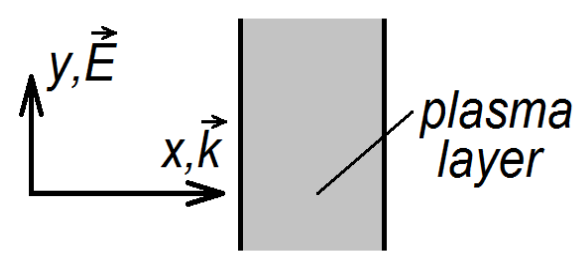

Fig. 1. To the model description

The following parameters were used in the simulation: plasma density $-4 \cdot 10^{12} \mathrm{~cm}^{-3}$ (electron plasma frequency - $18 \mathrm{GHz}$ ); plasma temperature $0.5 \mathrm{eV}$ (isothermal plasma); the plasma layer thickness $-46 \mathrm{~cm}$; incident wave length $-3.33 \mathrm{~cm}$ (frequency $9 \mathrm{GHz}$, i.e. plasma is opaque for this wave); pulse duration - $7.1 \mathrm{~ns}$; pulse width (at half maximum) $1.93 \cdot 10^{6} \mathrm{~V} / \mathrm{cm}$ (weak wave) and $7.73 \cdot 10^{6} \mathrm{~V} / \mathrm{cm}$ (strong wave). 


\section{SIMULATION RESULTS}

Figs. 2, 3 present the simulation results for a different incident electromagnetic pulse power and different time points. All the figures in the upper row show the spatial distributions of absolute values of electric field $E_{y}$ (left) and current density (right), in the lower row - the spatial distributions of electrons` (left) and ions` (right) density.

Figs. 2,a,b shows the results for a weak wave at different time points. It is clear that the incident electromagnetic wave interaction with plasma
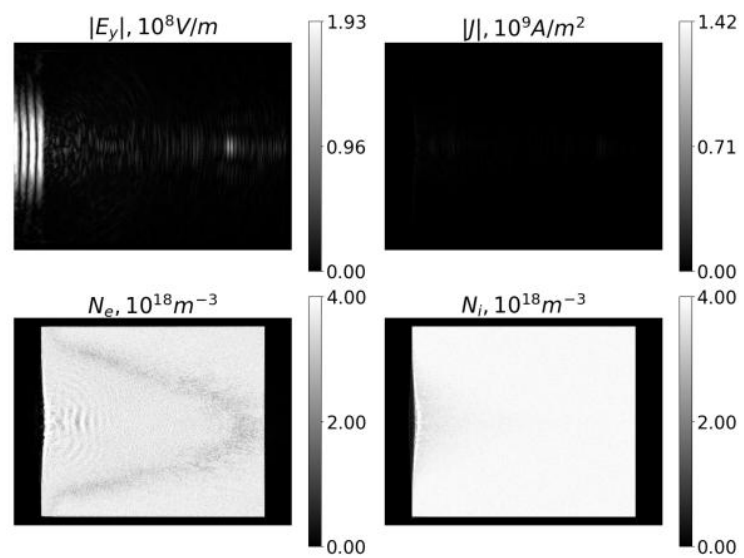

significantly distorts its field distribution (see Figs. 2,a,b). Fig. 2, a also shows a short electromagnetic pulse, which at this time point passed through the most part of the barrier. It can be associated with the front edge of the incident wave. The latter excites a highfrequency wave in the plasma, for which the barrier is transparent. The current flows both at the distorted ionic profile boundary (here it can be associated with a violation of electroneutrality due to the ponderomotive force) and in the depth of the plasma (see Fig. 2,b).

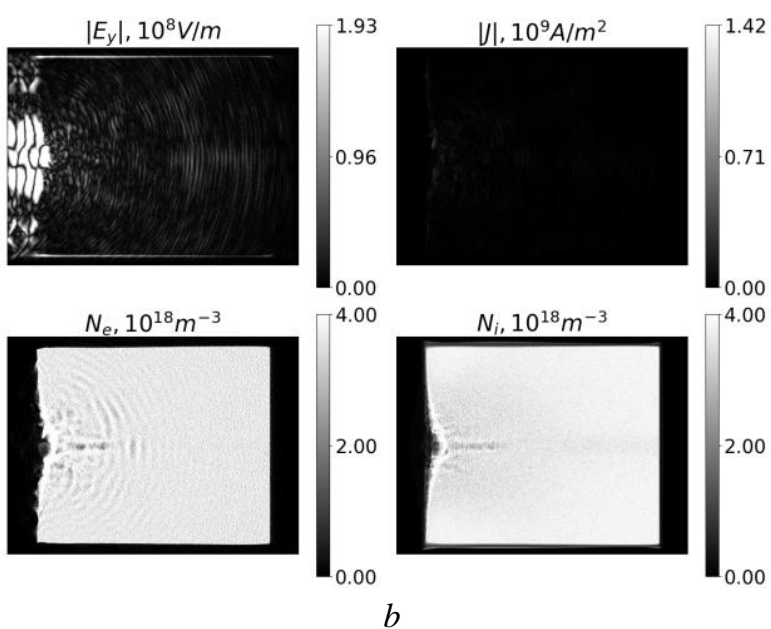

Fig. 2. Simulation results for a weak wave at the time points $2.63 \mathrm{~ns}(\mathrm{a})$ and $5.31 \mathrm{~ns}(\mathrm{~b})$
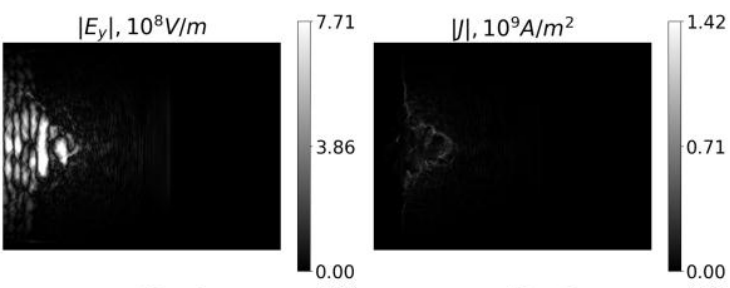

$N_{e}, 10^{18} \mathrm{~m}^{-3}$

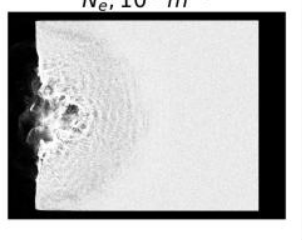

$7^{4.00} 2.00$

$N_{i}, 10^{18} \mathrm{~m}^{-3}$

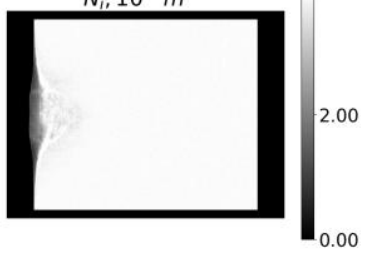

$a$
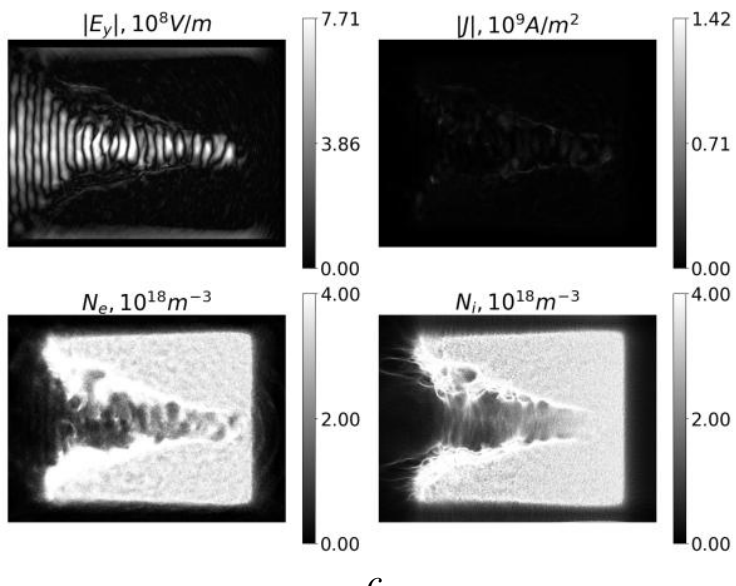
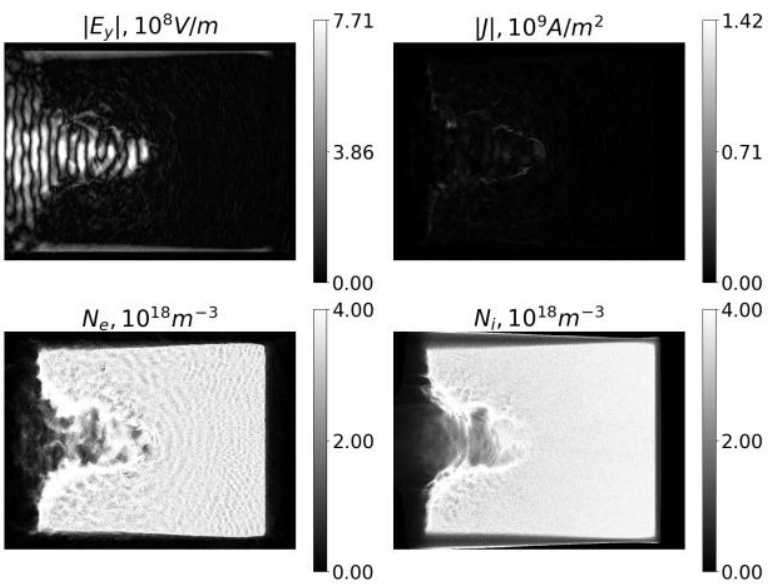

$b$
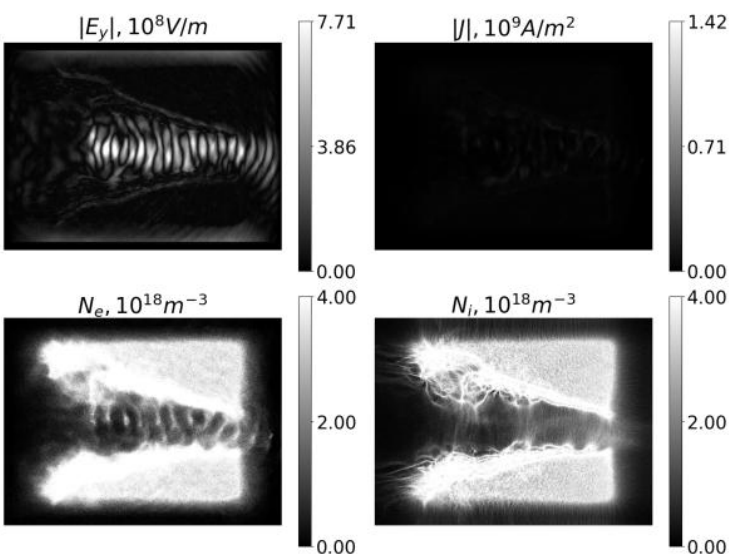

4.00
2.00
0.00

$N_{i}, 10^{18} m^{-3}$

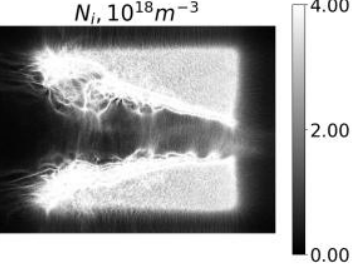

$d$

Fig. 3. Simulation results for a strong wave at time point $1.27 \mathrm{~ns}(a), 3.54 \mathrm{~ns}(\mathrm{~b}), 5.82 \mathrm{~ns}(\mathrm{c}), 7.69 \mathrm{~ns}(\mathrm{~d})$ 
The electron density distribution shows that perturbation propagates from the region of the electromagnetic pulse incidence into the plasma. In Figs. 2,a,b Langmuir waves are clearly visible. Fig. 2,a demonstrates three waves that propagate along the $x$ axis and up and down at equal angles to this axis. In Fig. 2,b wave fronts are similar to fragments of the concentric circles. The left border of electron gas is significantly distorted during the electromagnetic pulse action. It can be interpreted as the initial stage of the waveguide channel formation. One can expect that the channel will further deepen into plasma with the pulse duration increase. At the pulse back front, the channel edge is smeared. In Fig. 2,b the thin channel deepened into plasma along the $x$-axis is also visible clearly. But the density decrease in this channel is much smaller than in the deformed plasma boundary.

Changes in the ion density distribution are generally similar to the changes in electron density, but smallscale perturbations are less noticeable (at least initially). At Fig. 2,a the main ion gas volume still remains undisturbed, but at Fig. 2,b such perturbations are already clearly visible. Deviation from the symmetry of the upper and lower halves of the spatial distribution is noticeable in the electrons and ions density distributions.

Figs. 3,a-d shows the simulation results for a strong wave at different time points. It is seen that already in the initial time points (Figs. 3,a,b) the electron fluid motion becomes turbulent, the vortices are formed. As a result, areas of high and sharply reduced density with the scale of the order of the incident wave length are formed in it. Fig. 3,b shows that the similar ion density perturbations become noticeable.

The distributions of electron and ion densities in Fig. 3,c characterize the final stage of the waveguide channel formation in plasma. At the same time the initial symmetry concerning the middle plane $y=0$ is strongly disturbed. This effect can be preliminarily associated with the plasma instability in the field of an incident wave. In addition, the branching of the channel takes place in some cases. Finally, in Fig. 3,d one can see the already formed channels permeating the barrier. Note that during the channel formation currents flow in its entire volume.

As shown in Figs. 3,c-d a longitudinal quasiperiodic ion and electron density inhomogeneities are formed along the channel. The mechanism of their formation can be associated with the partial incident wave reflection and the standing wave formation in the channel. This is confirmed by the distributions of the electric field intensity, which correspond to standing waves.

Figs. 3,c-d also shows that the channel narrows in the incident wave vector direction. This effect can be explained by the fact that at the observation time, the ponderomotive force action time on the channel boundaries at its beginning is significantly longer than at its back part.

The channel formation is accompanied by the selffocusing of the incident electromagnetic wave (Figs. 3,a-d).

\section{DISCUSSION}

Let's compare the simulation results with the proposed theoretical mechanisms of the waveguide channel formation in the plasma barrier, described in the Introduction.

First of all, in the simulation we didn't observe excitation of Langmuir waves running from the electromagnetic beam incidence point in the plane parallel to the plasma surface. Therefore, the mechanisms associated with these waves are not confirmed by our simulation. However, it is possible to assume that plasma heating in the incident beam field by its electric field can contribute to "slow" transillumination effect together with the ponderomotive force. On the other hand, for the weak incident wave beam, Langmuir waves were observed with fronts close to spherical, propagating deep into plasma from the beam incidence point (see Fig. 2,b).

As already noted, for a strong incident wave beam quasi-periodic plasma inhomogeneity along the channel was observed at the late time points (see Figs. 3,c-d), similar to that predicted in [5]. However, this inhomogeneity occurs not at the initial stage but at the final stage of the channel formation and, therefore, is not associated with the "fast" transillumination.

Existence of the channel in the barrier after the wave pulse finish was also observed in the simulation. This effect is also consistent with the experimental results $[13,14]$.

Thus, the simulation results confirm the existence of only the first mechanism of the four theoretically predicted ones mentioned in the Introduction.

At the same time, the simulation results for the strong wave are in a good agreement with the considerations expressed in [12-14] regarding turbulence as a reason for the formation of small-scale (compared to the final channel size) inhomogeneities at the "fast" transillumination stage.

\section{CONCLUSIONS}

1. Transillumination of a dense plasma barrier by a strong electromagnetic wave beam has been simulated using the PIConGPU software package.

2. The simulation results correspond to the existing ideas about the mechanisms of "slow" transillumination (gradual formation of a waveguide channel with substantially reduced plasma density), but contradict all theoretically proposed mechanisms of "fast" transillumination.

3. Based on the simulation results, the cause for the formation of small-scale plasma inhomogeneities at the "fast" transillumination stage is the turbulent dynamics of the plasma in the field of the incident electromagnetic wave.

\section{REFERENCES}

1. I.O. Anisimov, L.I. Romaniuk. Transillumination, quasi-transparency and information transparency of the wave barriers in the inhomogeneous plasmas // Ukr. Phys. Journ. Reviews. 2010, v. 6, № 2, p. 101-139.

2. R. Betti, O.A. Hurricane. Inertial-confinement fusion with lasers // Nature Physics. 2016, v. 12, p. 435-448. 
3. A.G. Litvak. Dynamic non-linear electromagnetic phenomena in plasmas // Problems of Plasma Theory. Issue 10. M.: “Atomizdat”, 1980, p. 164-242.

4. G.M. Batanov, V.A. Silin. Non-linear penetration of the strong microwave field into the layer of dense collisionless plasma // Transactions of Lebedev FIAN. 1974, v. 73, p. 87-103.

5. A.G. Litvak. Self-focusing and waveguide propagation in plasmas // Physics-Uspekhi. 1972, v. 107 , № 3, p. 515-516 (in Russian).

6. A.G. Litvak, V.A. Mironov, G.M. Fraiman. On the self-action of electromagnetic waves in plasmas at the modulation instability // JETP Letters. 1975, v. 22, №7, p. 368-371.

7. G.M. Batanov, V.A. Silin. Self-action of electromagnetic wave in the dense collisionless plasma. // JETP Letters. 1971, v. 14, № 8, p. 445-448.

8. Yu.Ya. Brodskiy, B.G. Yeremin, A.G. Litvak, Yu.A. Sakhonchik. Self-canalization of the strong electromagnetic waves in the dense plasma. // JETP Letters. 1971, v. 13, № 3, p. 136-139.

9. M.I. Brizhinev, B.G. Yeremin, A.V. Kostrov, A.D. Ste-panushkin, G.M. Fraiman. Thermal transillumination of the magnetized plasma by the electromagnetic wave // Fizika plazmy. 1980, v. 6, № 3, p. 559-564 (in Russian).

10. B.G. Yeremin, A.V. Kostrov, A.D. Stepanushkin, G.M. Fraiman. Parametric heating of electrons in the HF field of the millimeter waveband // Fizika plazmy. 1976, v. 2, № 3, p. 414 (in Russian).
11. Yu.Ya. Brodskiy, V.L. Goltsman, V.A. Mironov, S.I. Nechuyev. Experimental study of electromagnetic field interaction with the plasma layer. 1978, v. 74, № 5, p. 1636-1649.

12. G.M. Batanov, Yu.V. Zadiraka, S.M. Levitsky, V.A. Silin, V.A. Tsygankov. Non-linear penetration of electromagnetic waves of various polarization into the dense collisionless plasma: FIAN preprint. 1979, v. 160 , p. 20.

13. G.M. Batanov, Yu.V. Zadiraka, S.M. Levitsky, S.N. Satunin, V.A. Silin, L.V. Sin'kovskaya. Redistribution of charges density due to the action of the strong electromagnetic wave of the microwave band on the dense collisionless plasma // All-Union conf. on the gaseous discharge physics. 1981, Part 1, p. 74-76.

14. G.M. Batanov, S.N. Satunin, V.A. Silin, L.V. Sin'kovskaya, Yu.V. Zadiraka, S.M. Levitsky. Density variation in the plasma layer due to the nonlinear transparency caused by the field of the strong electromagnetic wave // International Conference on Plasma Physics. Proceedings, Contributed Papers. Kiev, 1987, v. 1, p. 188-192.

15. M. Bussmann, H. Burau, T.E. Cowan, A. Debus, A. Huebl, G. Juckeland, T. Kluge, W.E. Nagel, R. Pausch, F. Schmitt, U. Schramm, J. Schuchart, R. Widera. Radiative signatures of the relativistic Kelvin-Helmholtz instability // SC'13 Proc. Int. Conf. High Perform. Comput. Networking, Storage Anal. 2013, v. 60, № 4, p. 1-12.

Article received 18.10 .2020

\title{
ПРОСВЕТЛЕНИЕ БАРЬЕРА ПЛОТНОЙ ПЛАЗМЫ МОЩНЫМ ПУЧКОМ ЭЛЕКТРОМАГНИТНЫХ ВОЛН: КОМПЬЮТЕРНОЕ МОДЕЛИРОВАНИЕ
}

\author{
Б.Р. Михайленко, И.А. Анисимов
}

Путем численного моделирования с применением программного пакета PIConGPU исследован процесс просветления барьера плотной плазмы сильным пучком электромагнитных волн. Наблюдалось формирование в плазме канала с пониженной плотностью, предсказанное теоретически и зафиксированное экспериментально. Для большей мощности падающего пучка наблюдалась турбулентная динамика плазмы, которая приводит к ее "быстрому" просветлению. Результаты моделирования не подтвердили предложенные теоретически механизмы "быстрого" просветления.

\section{ПРОСВІТЛЕННЯ БАР'СРА ЩІЛЬНӦ̈ ПЛАЗМИ ПОТУЖНИМ ПУЧКОМ ЕЛЕКТРОМАГНІТНИХ ХВИЛЬ: КОМП'ЮТЕРНЕ МОДЕЛЮВАННЯ}

\section{Б.Р. Михайленко, І.О. Анісімов}

Шляхом числового моделювання із застосуванням пакета PIConGPU досліджено процес просвітлення бар'єра щільної плазми сильним пучком електромагнітних хвиль. Спостерігалося формування в плазмі каналу зі зниженою густиною, передбачене теоретично і зафіксоване експериментально. Для більшої потужності падаючого пучка спостерігалася турбулентна динаміка плазми, яка призводить до їі "швидкого" просвітлення. Результати моделювання не підтвердили запропоновані теоретично механізми "швидкого" просвітлення. 